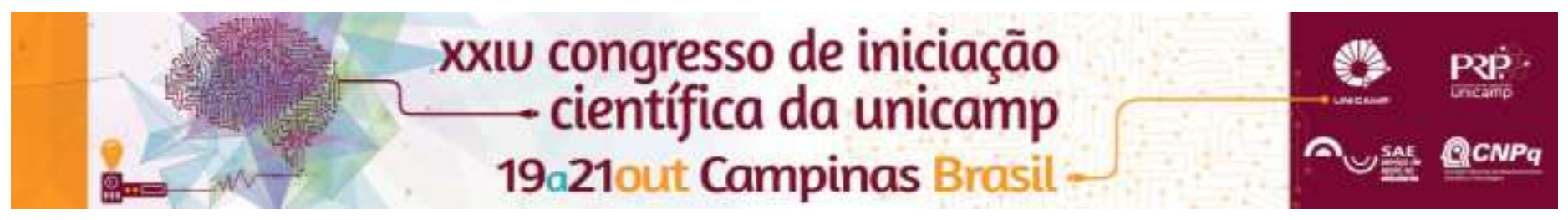

\title{
Comparação do Perfil Hemolitíco e Inflamatório em Doenças Hemolitícas.
}

\author{
Fernanda C.Z Fabris*, Flávia C. Leonardo, Kleber Y. Fertrin, Rodolfo D. Cançado, Sara T.O. Saad, Fernando F. \\ Costa, Nicola Conran, Camila B. Almeida.
}

\section{Resumo}

Nas anemias hemolíticas, um grupo de doenças em que a sobrevida do eritrócito na circulação é reduzida, pode ocorrer a liberação da hemoglobina $(\mathrm{Hb})$ no plasma. Doenças como Anemia Falciforme (AF), Esferocitose Hereditária $(E H)$ e Hemoglobinúria Paroxística Noturna (HPN) compartilham complicações geradas pelo excesso de Hb livre no plasma. Assim torna-se importante a melhor compreensão do processo hemolítico e suas consequências neste grupo de patologias.

\section{Palavras-chave:}

Hemólise; inflamação; heme

\section{Introdução}

AF, EH e HPN apresentam recorrentes eventos hemolíticos. Em situações de hemólise, a hemoglobina (Hb) e o grupamento heme são liberados no plasma resultando em depleção do óxido nítrico e inflamação. Embora essas doenças apresentem sintomas particulares, há indícios de que compartilhem complicações geradas pela hemólise. Todavia são escassos os estudos que investigam características do perfil hemolítico nestas doenças. Diante disso, nosso objetivo foi comparar o perfil hemolítico e inflamatório em pacientes com AF, AF em uso de hidroxiuréia (AFHU), EH, EH esplenectomizados (EHS) e HPN, através da quantificação de marcadores hemolíticos e inflamatórios.

\section{Métodos}

Sangue total foi coletado de indivíduos saudáveis (CON) e pacientes do Centro de Hematologia e Hemoterapia da Unicamp com as diferentes patologias. Os níveis de $\mathrm{Hb}$ livre $(\mathrm{HbL})$, heme, fator de necrose tumoral (TNF- $\alpha)$, haptoglobina $(\mathrm{Hp})$ e hemopexina $(\mathrm{Hpx})$ foram quantificados por ensaio colorimétrico ou ELISA. Níveis de bilirrubina indireta $(\mathrm{BI})$ e lactato desidrogenase (LDH) foram quantificados por método colorimétrico em sistema de automação modular (Roche AG Diagnostics).

\section{Resultados e Discussão}

Altos níveis de HbL, heme, LDH, BI, TNF- $\alpha$ e baixo nível de $\mathrm{Hp}$ observados nos pacientes AF e AFHU apoiam a hipótese de $\mathrm{HI}$ grave, a qual contribui para a inflamação presente na AF. Pacientes EH apresentaram níveis elevados de $\mathrm{BI}$ e TNF- $\alpha$, entretanto, não foi observado um aumento significativo de $\mathrm{HbL}$, heme e LDH, sugerindo que nesta patologia ocorra hemólise principalmente extra vascular (HE). Pacientes EHS não apresentaram níveis elevados de $\mathrm{Bl}$, mantiveram níveis elevados de TNF- $\alpha$ e apresentaram valores reduzidos de Hpx. Diante disso, pode-se sugerir que a inflamação nestes pacientes pode não ter uma relação direta com o processo hemolítico e que embora a esplenectomia possa colaborar para a diminuição da $\mathrm{HE}$ na $\mathrm{EH}$, existem particularidades, ainda pouco exploradas, na neutralização do heme e $\mathrm{HbL}$ liberados durante a $\mathrm{HI}$. Com relação à HPN, foram encontrados níveis elevados de $\mathrm{LDH}$ e intermediários dos demais marcadores, sugerindo que nesta doença ocorra uma hemólise mais branda. Além disso, não é possível afirmar que o valor de $\mathrm{LDH}$ encontrados estejam relacionados à $\mathrm{HI}$, pois muitos pacientes exibem complicações, o que pode ter influenciado o aumento dos níveis deste marcador.

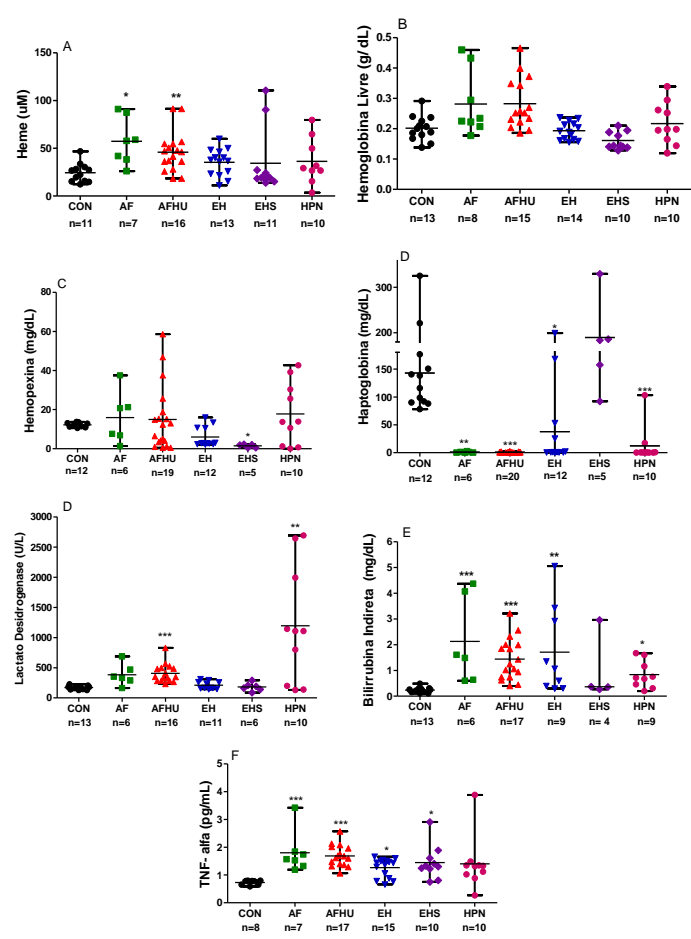

Figura 1. Quantificação de heme (A), HbL (B), Hpx (C), Hp (D), LDH (E), B (F) e TNF- $\alpha(G)$ de indivíduos CON, AF, AFHU, EHS e HPN. Os resultados estão expressos como média e desvio padrão. ${ }^{*} \mathrm{P}<0,05 ;{ }^{*} \mathrm{P}<0,01$ ${ }^{* \star *} \mathrm{P}<0,001$, comparado ao grupo CON, determinado por Kruskal Wallis, seguido pelo do teste de Dunn.

\section{Conclusões}

Neste estudo encontramos indícios de que o heme, $\mathrm{HbL}$ e $\mathrm{Hp}$ possam ser excelentes marcadores de $\mathrm{HI}$, enquanto que a $\mathrm{LDH}$ e $\mathrm{BI}$ devem estar associados a outros marcadores para um correto diagnóstico da hemólise.

\section{Agradecimentos}

\section{- JaPESP 나 o}

${ }^{1}$ Rother RP, Bell L, Hillmen P, Gladwin MT. The clinical sequelae of intravascular hemolysis and extracellular plasma hemoglobin: a novel mechanism of human disease. JAMA 2005; 293: 1653-1662. 